\title{
Remarks on the Avron-Herbst Type Formula for $N$-body Quantum Systems in Constant Electric and Magnetic Fields
}

\author{
Tadayoshi Adachi ${ }^{1 *}$ and Amane Kiyose ${ }^{2}$ \\ ${ }^{1}$ Graduate School of Human and Environmental Studies, Kyoto University, Japan \\ ${ }^{2}$ Department of Mathematics, Graduate School of Science, Kobe University, Japan \\ Email: adachi@math.h.kyoto-u.ac.jp
}

\begin{abstract}
In this paper, for $N \geq 2$, we give a natural derivation of the Avron-Herbst type formula for the time evolution generated by an $N$-body Hamiltonian with constant electric and magnetic fields. By virtue of the formula, some scattering problems can be reduced to those in the case where the constant electric and magnetic fields are parallel to each other. As an application of the formula, we give the result of the asymptotic completeness for the systems which have the only charged particle and some neutral ones in crossed constant electric and magnetic fields.
\end{abstract}

Keywords: Avron-Herbst type formula, constant electric field, constant magnetic field, time evolution.

\section{Introduction}

In this paper, we study the scattering theory for $N$-body quantum systems in constant electric and magnetic fields.

Let $N \geq 2$. Consider the system of $N$ particles moving in the Euclidean space $\boldsymbol{R}^{3}$ on which the constant electric field $\boldsymbol{E}=\left(E_{1}, E_{2}, E_{3}\right) \in \boldsymbol{R}^{3} \backslash\{0\}$ and the constant magnetic field $\boldsymbol{B}=(0,0, B) \in \boldsymbol{R}^{3} \backslash\{0\}$ with $B>0$ are impressed. Denote by $m_{j}>0, q_{j} \in \boldsymbol{R}$ and $x_{j}=\left(x_{j, 1}, x_{j, 2}, x_{j, 3}\right) \in \boldsymbol{R}^{3}(j=1, \ldots, N)$ the mass, the charge, and the position of the $j$-th particle, respectively. We assume that for some $N_{\mathrm{c}} \in \boldsymbol{N}$ such that $N_{\mathrm{c}} \leq N$, the last $N_{\mathrm{c}}$ particles are charged and the rest are neutral. In other words, we suppose

$$
\left.q_{j} \neq 0 \quad \text { (if } j \geq N_{\mathrm{n}}+1\right), \quad q_{j}=0 \quad \text { (otherwise), }
$$

where $N_{\mathrm{n}}:=N-N_{\mathrm{c}} \geq 0$. Then the total Hamiltonian $\tilde{H}(\boldsymbol{E})$ of the system is defined by

$$
\begin{aligned}
& \tilde{H}(\boldsymbol{E})=\tilde{H}_{0}(\boldsymbol{E})+V, \\
& \tilde{H}_{0}(\boldsymbol{E})=\sum_{j=1}^{N}\left(\frac{1}{2 m_{j}}\left(p_{j}-q_{j} \boldsymbol{A}\left(x_{j}\right)\right)^{2}-q_{j} \boldsymbol{E} \cdot x_{j}\right), \\
& V=\sum_{1 \leq j<k \leq N} V_{j k}\left(x_{j}-x_{k}\right),
\end{aligned}
$$

on $L^{2}\left(\boldsymbol{R}^{3 \times N}\right)$, where $p_{j}=-i \nabla_{x_{j}}=\left(p_{j, 1}, p_{j, 2}, p_{j, 3}\right)$ is the canonical momentum of the $j$-th particle, $V_{j k}\left(x_{j}-x_{k}\right)$ 's are pair potentials, and $\boldsymbol{A}(r)$ is the vector potential associated with the magnetic field $\boldsymbol{B}$. In the symmetric gauge, $\boldsymbol{A}(r)$ is written as

$$
\boldsymbol{A}(r)=\frac{1}{2} \boldsymbol{B} \times r=\frac{B}{2}\left(-r_{2}, r_{1}, 0\right), \quad r=\left(r_{1}, r_{2}, r_{3}\right) \in \boldsymbol{R}^{3} .
$$

We will use the symmetric gauge in this paper. Put

$$
D_{j}:=p_{j}-q_{j} \boldsymbol{A}\left(x_{j}\right)
$$


for the sake of brevity. $D_{j}$ is called the kinetic momentum of the $j$-th particle. Here we note that if $j \leq N_{\mathrm{n}}$, then $p_{j}=D_{j}$ because of $q_{j}=0$. For the sake of simplicity, we impose the following condition $(V 0)_{d}$ with $d=3$ on $V$ at first:

$(V 0)_{d}$ For $1 \leq j<k \leq N, V_{j k}$ belongs to $C\left(\boldsymbol{R}^{d} ; \boldsymbol{R}\right)$, and satisfies the decaying condition

$$
\left|V_{j k}(r)\right| \leq C\langle r\rangle^{-\rho}
$$

with some $\rho>0$.

Here $\langle r\rangle=\sqrt{1+r^{2}}$. Under the condition $(V 0)_{3}, \tilde{H}(\boldsymbol{E})$ is self-adjoint.

Put $\boldsymbol{E}_{\perp}:=\left(E_{1}, E_{2}, 0\right) \perp \boldsymbol{B}$ and $\boldsymbol{E}_{\|}:=\left(0,0, E_{3}\right) \| \boldsymbol{B}$. Then $\boldsymbol{E}$ can be decomposed into the direct sum $\boldsymbol{E}_{\perp} \oplus \boldsymbol{E}_{\|}$. Now we would like to give the relation between $e^{-i t \tilde{H}(\boldsymbol{E})}$ and $e^{-i t \tilde{H}\left(\boldsymbol{E}_{\|}\right)}$in terms of the Avron-Herbst type formula. Let us introduce the total mass $M$, the total charge $Q$, the position of the center of mass $x_{\mathrm{cm}}$, the total pseudomomentum $k_{\text {total }}$ of the system, and the $\boldsymbol{E} \times \boldsymbol{B}$ drift velocity $\alpha$ by

$$
\begin{aligned}
& M=\sum_{j=1}^{N} m_{j}, \quad Q=\sum_{j=1}^{N} q_{j}, \quad x_{\mathrm{cm}}=\frac{1}{M} \sum_{j=1}^{N} m_{j} x_{j}, \\
& k_{\text {total }}=\sum_{j=1}^{N}\left(p_{j}+q_{j} \boldsymbol{A}\left(x_{j}\right)\right), \quad \alpha=\frac{\boldsymbol{E} \times \boldsymbol{B}}{\boldsymbol{B}^{2}}=\left(\frac{E_{2}}{B},-\frac{E_{1}}{B}, 0\right) .
\end{aligned}
$$

Put

$$
k_{j}:=p_{j}+q_{j} \boldsymbol{A}\left(x_{j}\right)
$$

for the sake of brevity. $k_{j}$ is called the pseudomomentum of the $j$-th particle. Here we note that if $j \leq N_{\mathrm{n}}$, then $p_{j}=k_{j}$ because of $q_{j}=0$, and that

$$
k_{\text {total }}=\sum_{j=1}^{N} k_{j}
$$

holds. Then we obtain the following Avron-Herbst type formula for $e^{-i t \tilde{H}(\boldsymbol{E})}$ :

Theorem 1 Assume $V$ satisfies $(V 0)_{3}$. Then the Avron-Herbst type formula for $e^{-i t \tilde{H}(\boldsymbol{E})}$

$$
\begin{aligned}
& e^{-i t \tilde{H}(\boldsymbol{E})}=\tilde{\mathscr{T}}(t) e^{-i t \tilde{H}\left(\boldsymbol{E}_{\|}\right) \tilde{\mathscr{T}}(0)^{*}}, \\
& \tilde{\mathscr{T}}(t)=e^{-i t M \alpha^{2} / 2} e^{i M \alpha \cdot x_{\mathrm{cm}}} e^{-i t \alpha \cdot k_{\mathrm{total}}}
\end{aligned}
$$

holds.

We note $\tilde{\mathscr{T}}(0)=e^{i M \alpha \cdot x_{\mathrm{cm}}} \cdot e^{-i t \alpha \cdot k_{\text {total }}}$ in the definition of $\tilde{\mathscr{T}}(t)$ is called a magnetic translation generated by $k_{\text {total }}$. It is well-known that $e^{-i t \alpha \cdot k_{\text {total }}}$ can be written as

$$
e^{-i t \alpha \cdot k_{\mathrm{total}}}=e^{-i t \alpha \cdot \boldsymbol{A}\left(\tilde{x}_{\mathrm{cc}}\right)} e^{-i t \alpha \cdot p_{\mathrm{total}}}
$$

(see e.g. [1]), where $\tilde{x}_{\mathrm{cc}}$ and the total canonical momentum $p_{\text {total }}$ are given by

$$
\tilde{x}_{\mathrm{cc}}=\sum_{j=1}^{N} q_{j} x_{j}, \quad p_{\text {total }}=\sum_{j=1}^{N} p_{j} .
$$

If $Q \neq 0$, then the position of the center of charge $x_{\mathrm{cc}}$ can be given by

$$
x_{\mathrm{cc}}=\frac{1}{Q} \tilde{x}_{\mathrm{cc}},
$$

and (4) can be written as

$$
e^{-i t \alpha \cdot k_{\mathrm{total}}}=e^{-i t \alpha \cdot Q \boldsymbol{A}\left(x_{\mathrm{cc}}\right)} e^{-i t \alpha \cdot p_{\mathrm{total}}} .
$$


Hence $e^{-i t \alpha \cdot k_{\text {total }}}$ should be called a magnetic translation of the center of charge.

The Avron-Herbst type formula for $e^{-i t \tilde{H}(\boldsymbol{E})}$ like (3) was already obtained by Skibsted [2]. In fact, he introduced

$$
U_{1}(t)=\prod_{j=1}^{N} G_{j}(t), \quad G_{j}(t)=e^{i t m_{j} \alpha^{2} / 2} e^{-i t \alpha \cdot p_{j}} e^{i\left(t q_{j} \boldsymbol{A}(\alpha)+m_{j} \alpha\right) \cdot x_{j}},
$$

where $G_{j}(t)$ is the Galilei transform associated with the $j$-th particle which reflects the effect of the constant magnetic field $\boldsymbol{B}$. One of the basic properties of $G_{j}(t)$ is that

$$
G_{j}(t)^{*} x_{j} G_{j}(t)=x_{j}+t \alpha, \quad G_{j}(t)^{*} D_{j} G_{j}(t)=D_{j}+m_{j} \alpha
$$

hold. Then he claimed that the Avron-Herbst type formula

$$
e^{-i t \tilde{H}(\boldsymbol{E})}=U_{1}(t) e^{-i t \tilde{H}\left(\boldsymbol{E}_{\|}\right)} U_{1}(0)^{*}
$$

holds. Since charged particles drift with the $\boldsymbol{E} \times \boldsymbol{B}$ drift velocity $\alpha$, it is natural to consider the Galilei transform $G_{j}(t)$ for each charged particle. However, it is not certain whether the Galilei transforms $G_{j}(t)$ 's must be introduced also for neutral particles. Notice that neutral particles can move freely independent of the drift velocity $\alpha$. One of the purposes of this paper is to give a natural definition of an equivalent of $U_{1}(t)$ even if the system under consideration has some neutral particles, that is, $N_{\mathrm{n}} \geq 1$.

Also in the case where the space dimension $d$ is not three but two, the Avron-Herbst type formula can be obtained quite similarly: We suppose that the constant magnetic field $\boldsymbol{B}$ is perpendicular to the plane $\boldsymbol{R}^{2}$, and that the constant electric field $E=\left(E_{1}, E_{2}\right) \in \boldsymbol{R}^{2} \backslash\{0\}$ lies in the plane. We use the notation

$$
\begin{aligned}
& x_{j, \perp}=\left(x_{j, 1}, x_{j, 2}\right), \quad p_{j, \perp}=\left(p_{j, 1}, p_{j, 2}\right), \quad A\left(x_{j, \perp}\right)=\frac{B}{2}\left(-x_{j, 2}, x_{j, 1}\right), \\
& D_{j, \perp}=p_{j, \perp}-q_{j} A\left(x_{j, \perp}\right), \quad k_{j, \perp}=p_{j, \perp}+q_{j} A\left(x_{j, \perp}\right), \\
& x_{\mathrm{cm}, \perp}=\frac{1}{M} \sum_{j=1}^{N} m_{j} x_{j, \perp}, \quad \tilde{x}_{\mathrm{cc}, \perp}=\sum_{j=1}^{N} q_{j} x_{j, \perp}, \quad p_{\text {total }, \perp}=\sum_{j=1}^{N} p_{j, \perp}, \\
& D_{\text {total }, \perp}=\sum_{j=1}^{N} D_{j, \perp}=p_{\text {total }, \perp}-A\left(\tilde{x}_{\mathrm{cc}, \perp}\right), \\
& k_{\text {total }, \perp}=\sum_{j=1}^{N} k_{j, \perp}=p_{\text {total }, \perp}+A\left(\tilde{x}_{\mathrm{cc}, \perp}\right) .
\end{aligned}
$$

Then the total Hamiltonian $\tilde{H}_{\perp}(E)$ of the system is defined by

$$
\begin{aligned}
& \tilde{H}_{\perp}(E)=\tilde{H}_{0, \perp}(E)+V, \\
& \tilde{H}_{0, \perp}(E)=\sum_{j=1}^{N}\left(\frac{1}{2 m_{j}} D_{j, \perp}^{2}-q_{j} E \cdot x_{j, \perp}\right), \\
& V=\sum_{1 \leq j<k \leq N} V_{j k}\left(x_{j, \perp}-x_{k, \perp}\right) .
\end{aligned}
$$

on $L^{2}\left(\boldsymbol{R}^{2 \times N}\right)$. Under the condition $(V 0)_{2}, \tilde{H}_{\perp}(E)$ is self-adjoint. Then we obtain the following AvronHerbst type formula for $e^{-i t \tilde{H}_{\perp}(E)}$ :

Theorem 2 Assume $V$ satisfies $(V 0)_{2}$. Then the Avron-Herbst type formula for $e^{-i t \tilde{H}_{\perp}(E)}$

$$
\begin{aligned}
& e^{-i t \tilde{H}_{\perp}(E)}=\tilde{\mathscr{T}}_{\perp}(t) e^{-i t \tilde{H}_{\perp}(0)} \tilde{\mathscr{T}}_{\perp}(0)^{*} \\
& \tilde{\mathscr{T}}_{\perp}(t)=e^{-i t M \alpha_{\perp}^{2} / 2} e^{i M \alpha_{\perp} \cdot x_{\mathrm{cm}, \perp}} e^{-i t \alpha_{\perp} \cdot k_{\mathrm{total}, \perp}}
\end{aligned}
$$

holds. 
Since $\alpha=\left(\alpha_{\perp}, 0\right), \tilde{\mathscr{T}}(t)$ in Theorem 1 can be represented as

$$
\tilde{\mathscr{T}}(t)=\tilde{\mathscr{T}}_{\perp}(t) \otimes \mathrm{Id}
$$

on $L^{2}\left(\boldsymbol{R}^{3 \times N}\right) \cong L^{2}\left(\boldsymbol{R}^{2 \times N}\right) \otimes L^{2}\left(\boldsymbol{R}^{N}\right)$. Hence we have only to show Theorem 2 essentially. We will give the proof in $\S 2$.

When $N=1$, the Avron-Herbst type formula for the free propagator was already obtained by AdachiKawamoto [3], even if the homogeneous electric field is strictly time-dependent. Here we note that before the work [3], a different but meaningful factorization of the free propagator was given by Chee [4]. In the case where the homogeneous electric field is constant, as for some spectral problems for perturbed Hamiltonians, see Wang [5], Dimassi-Petkov [6], [7], [8], Ferrari-Kovař́k [9], [10], and Kawamoto [11]; while in the homogeneous electric field is time-dependent, Lawson and Avossevou [12] have recently studied a certain spectral problem for the free Hamiltonian with time-dependent mass (see also the references therein).

On the other hand, when $N \geq 2$, in general, it seems hard to obtain a certain effective Avron-Herbst type formula if the homogeneous electric field is time-dependent, except in the case where all the specific charges of particles are the same, that is, $x_{\mathrm{cm}}=x_{\mathrm{cc}}$. We will mention it in $\S 4$.

The structure of this paper is as follows: In $\S 2$, we will give the proof of Theorem 2 . In $\S 3$, as an application of our results, we will deal with the problem of the asymptotic completeness for the systems which have the only charged particle and some neutral ones in crossed constant electric and magnetic fields, mainly in the short-range case. In $§ 4$, we will make some remarks on the extension to the case where the homogeneous electric field is strictly time-dependent.

\section{Proof of Theorem 2}

In this section, we will show Theorems 1 and 2. As mentioned in $\S 1$, we have only to give the proof of Theorem 2.

First of all, we note that

$$
\begin{aligned}
& k_{\text {total }, \perp}-D_{\text {total }, \perp}=2 \sum_{j=1}^{N} q_{j} A\left(x_{j, \perp}\right)=2 A\left(\tilde{x}_{\mathrm{cc}, \perp}\right), \\
& A\left(A\left(r_{\perp}\right)\right)=-\left(\frac{B}{2}\right)^{2} r_{\perp}, \quad \hat{r}_{\perp} \cdot A\left(r_{\perp}\right)=-A\left(\hat{r}_{\perp}\right) \cdot r_{\perp}
\end{aligned}
$$

hold for $r_{\perp}, \hat{r}_{\perp} \in \boldsymbol{R}^{2}$. Then $\tilde{H}_{0, \perp}(E)$ can be represented as

$$
\begin{aligned}
\tilde{H}_{0, \perp}(E) & =\sum_{j=1}^{N} \frac{1}{2 m_{j}} D_{j, \perp}^{2}-E \cdot \tilde{x}_{\mathrm{cc}, \perp} \\
& =\sum_{j=1}^{N} \frac{1}{2 m_{j}} D_{j, \perp}^{2}+\frac{2}{B^{2}} E \cdot A\left(k_{\mathrm{total}, \perp}-D_{\mathrm{total}, \perp}\right) \\
& =\sum_{j=1}^{N} \frac{1}{2 m_{j}} D_{j, \perp}^{2}-\frac{2}{B} A\left(\frac{E}{B}\right) \cdot\left(k_{\mathrm{total}, \perp}-D_{\mathrm{total}, \perp}\right) .
\end{aligned}
$$

Noticing

$$
\alpha_{\perp}=-\frac{2}{B} A\left(\frac{E}{B}\right)
$$

we have

$$
\tilde{H}_{0, \perp}(E)=\sum_{j=1}^{N} \frac{1}{2 m_{j}} D_{j, \perp}^{2}+\alpha_{\perp} \cdot\left(k_{\mathrm{total}, \perp}-D_{\mathrm{total}, \perp}\right)
$$




$$
=\sum_{j=1}^{N} \frac{1}{2 m_{j}}\left(D_{j, \perp}-m_{j} \alpha_{\perp}\right)^{2}+\alpha_{\perp} \cdot k_{\mathrm{total}, \perp}-\frac{M}{2} \alpha_{\perp}^{2} .
$$

Putting

$$
\tilde{T}_{\perp}:=\sum_{j=1}^{N} \frac{1}{2 m_{j}}\left(D_{j, \perp}-m_{j} \alpha_{\perp}\right)^{2}+V,
$$

we see that $\tilde{T}_{\perp}$ does commute with $\alpha_{\perp} \cdot k_{\text {total, } \perp \text {, and that }}$

$$
\tilde{T}_{\perp}=e^{i M \alpha_{\perp} \cdot x_{\mathrm{cm}, \perp}} \tilde{H}_{\perp}(0) e^{-i M \alpha_{\perp} \cdot x_{\mathrm{cm}, \perp}}
$$

holds. Hence we have

$$
\begin{aligned}
e^{-i t \tilde{H}_{\perp}(E)} & =e^{i M t \alpha_{\perp}^{2} / 2} e^{-i t \alpha_{\perp} \cdot k_{\mathrm{total}, \perp}} e^{-i t \tilde{T}_{\perp}} \\
& =e^{i M t \alpha_{\perp}^{2} / 2} e^{-i t \alpha_{\perp} \cdot k_{\mathrm{total}, \perp}} e^{i M \alpha_{\perp} \cdot x_{\mathrm{cm}, \perp}} e^{-i t \tilde{H}_{\perp}(0)} e^{-i M \alpha_{\perp} \cdot x_{\mathrm{cm}, \perp}} \\
& =e^{-i t M \alpha_{\perp}^{2} / 2} e^{i M \alpha_{\perp} \cdot x_{\mathrm{cm}, \perp}} e^{-i t \alpha_{\perp} \cdot k_{\mathrm{total}, \perp}} e^{-i t \tilde{H}_{\perp}(0)} e^{-i M \alpha_{\perp} \cdot x_{\mathrm{cm}, \perp}}
\end{aligned}
$$

which yields (9). Thus the proof is completed.

\section{Application}

In this section, we will apply the Avron-Herbst type formula to some scattering problems for $N$-body quantum systems in constant electric and magnetic fields, which have neutral particles. In order to make the point at issue clear, we suppose that the space dimension $d$ is two. The case where $d=3$ and $\boldsymbol{E}_{\|}=0$ can be also dealt with quite similarly. The case where $d=3$ and $\boldsymbol{E}_{\|} \neq 0$ can be treated by the results due to Skibsted [2]. We impose the following condition $(V 1)_{2, \text { SR }}$ on $V$, which is stronger than $(V 0)_{2}$ :

$(V 1)_{2, \mathrm{SR}}$ For $1 \leq j<k \leq N, V_{j k}$ belongs to $C^{2}\left(\boldsymbol{R}^{2} ; \boldsymbol{R}\right)$, and satisfies the decaying condition

$$
\left|\partial_{r}^{\beta} V_{j k}(r)\right| \leq C_{\beta}\langle r\rangle^{-\rho-|\beta|}, \quad|\beta| \leq 2
$$

with some $\rho>1$.

We consider the problem of the asymptotic completeness for an $N$-body quantum system in the plane $\boldsymbol{R}^{2}$ to which constant electric and magnetic fields are impressed. Suppose $N_{\mathrm{c}}=1$ and $N_{\mathrm{n}}=N-1 \geq 1$. Then the total Hamiltonian $\tilde{H}_{\perp}(E)$ on $L^{2}\left(\boldsymbol{R}^{2 \times N}\right)$ is represented as

$$
\begin{aligned}
& \tilde{H}_{\perp}(E)=\tilde{H}_{0, \perp}(E)+V \\
& \tilde{H}_{0, \perp}(E)=\sum_{j=1}^{N-1} \frac{1}{2 m_{j}} p_{j, \perp}^{2}+\left(\frac{1}{2 m_{N}} D_{N, \perp}^{2}-q_{N} E \cdot x_{N, \perp}\right)
\end{aligned}
$$

with $q_{N} \neq 0 . \tilde{H}_{\perp}(E)$ has a pure absolutely continuous spectrum, that is,

$$
L_{\mathrm{ac}}^{2}\left(\tilde{H}_{\perp}(E)\right)=L^{2}\left(\boldsymbol{R}^{2 \times N}\right)
$$

where $L_{\text {ac }}^{2}\left(\tilde{H}_{\perp}(E)\right)$ is the absolutely continuous spectral subspace associated with $\tilde{H}_{\perp}(E)$. In fact, putting $\tilde{A}=q_{N} E \cdot k_{\text {total, } \perp}$ as in Adachi-Kawamoto [3],

$$
i\left[\tilde{H}_{\perp}(E), \tilde{A}\right]=q_{N}^{2} E^{2}>0
$$

holds even if $V \neq 0$, which implies the above property.

When $E=0$, the results of the asymptotic completeness for

$$
\tilde{H}_{\perp}(0)=\sum_{j=1}^{N-1} \frac{1}{2 m_{j}} p_{j, \perp}^{2}+\frac{1}{2 m_{N}} D_{N, \perp}^{2}+V
$$


were already obtained by Adachi [13] and [14]. We will show the asymptotic completeness for $\tilde{H}_{\perp}(E)$ by using those. For the sake of explanation, we introduce some notation in the many body scattering theory: A non-empty subset of the set $\{1, \ldots, N\}$ is called a cluster. Let $C_{j}, 1 \leq j \leq m$, be clusters. If $\cup_{1 \leq j \leq m} C_{j}=\{1, \ldots, N\}$ and $C_{j} \cap C_{k}=\emptyset$ for $1 \leq j<k \leq m$, then $a=\left\{C_{1}, \ldots, C_{m}\right\}$ is called a cluster decomposition. \# $(a)$ denotes the number of clusters in $a$. Let $\mathscr{A}$ be the set of all cluster decompositions. Suppose $a, b \in \mathscr{A}$. If $b$ is obtained as a refinement of $a$, that is, if each cluster in $b$ is a subset of a cluster in $a$, we say $b \subset a$, and its negation is denoted by $b \not \subset a$. Any $a$ is regarded as a refinement of itself. The one- and $N$-cluster decompositions are denoted by $a_{\max }$ and $a_{\min }$, respectively. The pair $(j, k)$ is identified with the $(N-1)$-cluster decomposition $\{(j, k),(1), \ldots,(\widehat{j}), \ldots,(\widehat{k}), \ldots,(N)\}$. For $a \in \mathscr{A}$, the cluster Hamiltonian $\tilde{H}_{a, \perp}(E)$ and the intercluster potential $I_{a}$ are defined by

$$
\begin{aligned}
& \tilde{H}_{a, \perp}(E)=\tilde{H}_{0, \perp}(E)+V^{a}, \quad V^{a}=\sum_{(j, k) \subset a} V_{j k}\left(x_{j, \perp}-x_{k, \perp}\right), \\
& I_{a}=V-V^{a}=\sum_{(j, k) \not \subset a} V_{j k}\left(x_{j, \perp}-x_{k, \perp}\right) .
\end{aligned}
$$

Here we note

$$
\tilde{H}_{a_{\max }, \perp}(E)=\tilde{H}_{\perp}(E), \quad \tilde{H}_{a_{\min }, \perp}(E)=\tilde{H}_{0, \perp}(E) .
$$

Of course, $\tilde{H}_{a, \perp}(0)$ can be defined similarly. Let $a=\left\{C_{1}, \ldots, C_{\#(a)}\right\} \in \mathscr{A}$. For the sake of simplicity, we suppose $N \in C_{\#(a)}$. For each cluster $C_{l}$ in $a$, the innercluster Hamiltonian $\tilde{H}_{\perp}^{C_{l}}(0)$ is defined by

$$
\tilde{H}_{\perp}^{C_{l}}(0)=\sum_{j \in C_{l}} \frac{1}{2 m_{j}} D_{j, \perp}^{2}+V^{C_{l}}, \quad V^{C_{l}}=\sum_{\{j, k\} \subset C_{l}} V_{j k}\left(x_{j, \perp}-x_{k, \perp}\right),
$$

on $L^{2}\left(\boldsymbol{R}^{2 \times \#\left(C_{l}\right)}\right)$, where $\#\left(C_{l}\right)$ denotes the number of elements in $C_{l}$. In particular, when $l=\#(a)$, $\tilde{H}_{\perp}^{C_{\#(a)}}(0)$ is represented as

$$
\tilde{H}_{\perp}^{C_{\#(a)}}(0)=\sum_{\substack{j \in C_{\#(a)} \\ j<N}} \frac{1}{2 m_{j}} p_{j, \perp}^{2}+\frac{1}{2 m_{N}} D_{N, \perp}^{2}+V^{C_{\#(a)}} .
$$

If $N=\#\left(C_{\#(a)}\right)$, that is, $a=a_{\max }$, then $\tilde{H}_{\perp}^{C_{\#(a)}}(0)$ is just equal to $\tilde{H}_{\perp}(0)$. On the other hand, when $l<\#(a)$,

$$
\tilde{H}_{\perp}^{C_{l}}(0)=\sum_{j \in C_{l}} \frac{1}{2 m_{j}} p_{j, \perp}^{2}+V^{C_{l}}
$$

is just a \# $\left(C_{l}\right)$-body Schrödinger operator without external electromagnetic fields. Here we would like to consider the sum of all the innercluster Hamiltonians except $\tilde{H}_{\perp}^{C_{\#(a)}}(0)$ in the center-of-mass frame: Firstly, we will equip $\boldsymbol{R}^{2 \times \#\left(C_{l}\right)}, l=1, \ldots, \#(a)-1$, with the metric

$$
\langle\eta, \tilde{\eta}\rangle=\sum_{k=1}^{\#\left(C_{l}\right)} m_{c_{l}(k)} x_{c_{l}(k), \perp} \cdot \tilde{x}_{c_{l}(k), \perp}
$$

for $\eta=\left(x_{c_{l}(1), \perp}, \ldots, x_{c_{l}\left(\#\left(C_{l}\right)\right), \perp}\right), \tilde{\eta}=\left(\tilde{x}_{c_{l}(1), \perp}, \ldots, \tilde{x}_{c_{l}\left(\#\left(C_{l}\right)\right), \perp}\right) \in \boldsymbol{R}^{2 \times \#\left(C_{l}\right)}$, and define two subspaces $X_{\perp}^{C_{l}}$ and $X_{C_{l}, \perp}$ of $\boldsymbol{R}^{2 \times \#\left(C_{l}\right)}$ by

$$
\begin{aligned}
& X_{\perp}^{C_{l}}=\left\{\left(x_{c_{l}(1), \perp}, \ldots, x_{c_{l}\left(\#\left(C_{l}\right)\right), \perp}\right) \in \boldsymbol{R}^{2 \times \#\left(C_{l}\right)} \mid \sum_{k=1}^{\#\left(C_{l}\right)} m_{c_{l}(k)} x_{c_{l}(k), \perp}=0\right\}, \\
& X_{C_{l}, \perp}=\boldsymbol{R}^{2 \times \#\left(C_{l}\right)} \ominus X_{\perp}^{C_{l}} .
\end{aligned}
$$

Secondly, we will define two subspaces $X_{\perp}^{a, \mathrm{n}}$ and $X_{a, \mathrm{n}, \perp}$ of $\boldsymbol{R}^{2 \times\left(N-\#\left(C_{\#(a)}\right)\right)}$ by

$$
X_{\perp}^{a, \mathrm{n}}=X_{\perp}^{C_{1}} \times \cdots \times X_{\perp}^{C_{\#(a)-1}}, \quad X_{a, \mathrm{n}, \perp}=\boldsymbol{R}^{2 \times\left(N-\#\left(C_{\#(a)}\right)\right)} \ominus X_{\perp}^{a, \mathrm{n}}
$$


which are equipped with the metric $\langle$,$\rangle . Then the sum of all the innercluster Hamiltonians except$ $\tilde{H}_{\perp}^{C \#(a)}(0)$ can be decomposed into

$$
\tilde{H}_{\perp}^{a, \mathrm{n}} \otimes \operatorname{Id}+\operatorname{Id} \otimes\left(-\frac{1}{2} \Delta_{X_{a, \mathrm{n}, \perp}}\right), \quad \tilde{H}_{\perp}^{a, \mathrm{n}}=-\frac{1}{2} \Delta_{X_{\perp}^{a, \mathrm{n}}}+\left(V^{a}-V^{C \#(a)}\right),
$$

on $L^{2}\left(\boldsymbol{R}^{2 \times\left(N-\#\left(C_{\#(a)}\right)\right)}\right) \cong L^{2}\left(X_{\perp}^{a, \mathrm{n}}\right) \otimes L^{2}\left(X_{a, \mathrm{n}, \perp}\right)$, where $\Delta_{X_{\perp}^{a, \mathrm{n}}}$ and $\Delta_{X_{a, \mathrm{n}, \perp}}$ are the Laplace-Beltrami operators on $X_{\perp}^{a, \mathrm{n}}$ and $X_{a, \mathrm{n}, \perp}$, respectively. $\tilde{H}_{\perp}^{a, \mathrm{n}}$ is an $\left(N-\#\left(C_{\#(a)}\right)\right)$-body Schrödinger operator without external electromagnetic fields in the center-of-mass frame. Thus we have

$$
\tilde{H}_{a, \perp}(0)=\tilde{H}_{\perp}^{a, \mathrm{n}} \otimes \operatorname{Id} \otimes \operatorname{Id}+\operatorname{Id} \otimes \tilde{H}_{\perp}^{C_{\#(a)}}(0) \otimes \operatorname{Id}+\operatorname{Id} \otimes \operatorname{Id} \otimes\left(-\frac{1}{2} \Delta_{X_{a, \mathrm{n}, \perp}}\right)
$$

on $L^{2}\left(\boldsymbol{R}^{2 \times N}\right) \cong L^{2}\left(X_{\perp}^{a, \mathrm{n}}\right) \otimes L^{2}\left(\boldsymbol{R}^{2 \times \#\left(C_{\#(a)}\right)}\right) \otimes L^{2}\left(X_{a, \mathrm{n}, \perp}\right)$. We put

$$
\Pi^{a}(0):=P_{\mathrm{pp}}\left(\tilde{H}_{\perp}^{a, \mathrm{n}}\right) \otimes P_{\mathrm{pp}}\left(\tilde{H}_{\perp}^{C \#(a)}(0)\right) \otimes \mathrm{Id}
$$

on $L^{2}\left(\boldsymbol{R}^{2 \times N}\right) \cong L^{2}\left(X_{\perp}^{a, \mathrm{n}}\right) \otimes L^{2}\left(\boldsymbol{R}^{2 \times \#\left(C_{\#(a)}\right)}\right) \otimes L^{2}\left(X_{a, \mathrm{n}, \perp}\right)$, where $P_{\mathrm{pp}}\left(\tilde{H}_{\perp}^{a, \mathrm{n}}\right)$ and $P_{\mathrm{pp}}\left(\tilde{H}_{\perp}^{C_{\#(a)}}(0)\right)$ are the eigenprojections for $\tilde{H}_{\perp}^{a, \mathrm{n}}$ and $\tilde{H}_{\perp}^{C_{\#(a)}}(0)$, respectively. If $a=a_{\max }$, then $\Pi^{a_{\max }}(0)=P_{\mathrm{pp}}\left(\tilde{H}_{\perp}(0)\right)$ holds; while, if $a=a_{\min }$, then $\Pi^{a_{\min }}(0)=\mathrm{Id}$ holds. Here we introduce the wave operators

$$
\begin{aligned}
& W_{a}^{ \pm}(E)=\underset{t \rightarrow \pm \infty}{\mathrm{s}-\lim } e^{i t \tilde{H}_{\perp}(E)} e^{-i t \tilde{H}_{a, \perp}(E)} \Pi^{a}(E), \\
& \Pi^{a}(E)=\tilde{\mathscr{T}}_{\perp}(0) \Pi^{a}(0) \tilde{\mathscr{T}}_{\perp}(0)^{*}=e^{i M \alpha_{\perp} \cdot x_{\mathrm{cm}, \perp}} \Pi^{a}(0) e^{-i M \alpha_{\perp} \cdot x_{\mathrm{cm}, \perp}},
\end{aligned}
$$

for $a \in \mathscr{A} . W_{a_{\max }}^{ \pm}(E)$ is identified with $\Pi^{a_{\max }}(E)$. Then one can obtain the following result of the asymptotic completeness for $\tilde{H}_{\perp}(E)$ :

Theorem 3 Assume $V$ satisfies $(V 1)_{2, \mathrm{SR}}$. Then the wave operators $W_{a}^{ \pm}(E), a \in \mathscr{A}$, all exist, and are asymptotically complete:

$$
L^{2}\left(\boldsymbol{R}^{2 \times N}\right)=\sum_{a \in \mathscr{A}} \oplus \operatorname{Ran} W_{a}^{ \pm}(E) .
$$

This theorem with $N=2$ was already obtained by Kiyose [15]. The asymptotic completeness (21) is equivalent to that the time evolution $e^{-i t \tilde{H}_{\perp}(E)} \psi$ of any scattering state $\psi \in L_{\text {ac }}^{2}\left(\tilde{H}_{\perp}(E)\right)=L^{2}\left(\boldsymbol{R}^{2 \times N}\right)$ is asymptotically represented as

$$
e^{-i t \tilde{H}_{\perp}(E)} \psi=\sum_{a \in \mathscr{A}} e^{-i t \tilde{H}_{a, \perp}(E)} \Pi^{a}(E) \psi_{a}^{ \pm}+o(1) \text { as } t \rightarrow \pm \infty
$$

with some $\psi_{a}^{ \pm} \in L^{2}\left(\boldsymbol{R}^{2 \times N}\right)$. In particular, $e^{-i t \tilde{H}_{a_{\max }, \perp}(E)} \Pi^{a_{\max }}(E) \psi_{a_{\max }}^{ \pm}$implies that all the particles in the system move with the velocity $\alpha_{\perp}$ in forming a certain bound state. In fact, the guiding center of the $N$-th particle, which is the only charged one, drifts with the velocity $\alpha_{\perp}$. This result can be obtained immediately by using Theorem 2 and the following result due to Adachi [13] and [14]:

Theorem 4 Assume $V$ satisfies $(V 1)_{2, \mathrm{SR}}$. Then the wave operators

$$
W_{a}^{ \pm}(0)=\underset{t \rightarrow \pm \infty}{s-\lim _{1}} e^{i t \tilde{H}_{\perp}(0)} e^{-i t \tilde{H}_{a, \perp}(0)} \Pi^{a}(0), \quad a \in \mathscr{A},
$$

all exist, and are asymptotically complete:

$$
L^{2}\left(\boldsymbol{R}^{2 \times N}\right)=\sum_{a \in \mathscr{A}} \oplus \operatorname{Ran} W_{a}^{ \pm}(0) .
$$


In fact, Theorem 2 yields

$$
W_{a}^{ \pm}(E)=\tilde{\mathscr{T}}_{\perp}(0) W_{a}^{ \pm}(0) \tilde{\mathscr{T}}_{\perp}(0)^{*}=e^{i M \alpha_{\perp} \cdot x_{\mathrm{cm}, \perp}} W_{a}^{ \pm}(0) e^{-i M \alpha_{\perp} \cdot x_{\mathrm{cm}, \perp}}
$$

for $a \in \mathscr{A}$. Hence, Theorem 3 can be obtained by virtue of Theorem 4 .

In the case where $N=2$, one can also obtain the result of the asymptotic completeness for $\tilde{H}_{\perp}(E)$ with some long-range potential $V=V_{12}$ by virtue of the result of [13]:

Theorem 5 Suppose $N=2$. Assume $V=V_{12}=I_{a_{\min }}$ belongs to $C^{\infty}\left(\boldsymbol{R}^{2} ; \boldsymbol{R}\right)$, and satisfies the decaying condition

$$
\left|\partial_{r}^{\beta} V(r)\right| \leq C_{\beta}\langle r\rangle^{-\rho-|\beta|}
$$

with some $1 / 2<\rho \leq 1$. Then the modified wave operators

$$
W_{a_{\min }, D}^{ \pm}(E)=\underset{t \rightarrow \pm \infty}{\mathrm{s}-\lim } e^{i t \tilde{H}_{\perp}(E)} e^{-i t \tilde{H}_{a_{\min }, \perp}(E)} e^{-i \int_{0}^{t} V\left(s p_{1, \perp} / m_{1}-s \alpha_{\perp}\right) d s},
$$

exist, and are asymptotically complete:

$$
L^{2}\left(\boldsymbol{R}^{2 \times 2}\right)=\operatorname{Ran} W_{a_{\min }, D}^{ \pm}(E) \oplus \operatorname{Ran} \Pi^{a_{\max }}(E) .
$$

By virtue of the result of [13], the modified wave operators

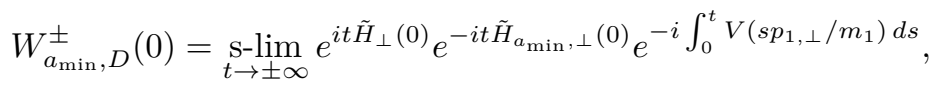

exist, and are asymptotically complete:

$$
L^{2}\left(\boldsymbol{R}^{2 \times 2}\right)=\operatorname{Ran} W_{a_{\min }, D}^{ \pm}(0) \oplus \operatorname{Ran} \Pi^{a_{\max }}(0) .
$$

Since Theorem 2 yields

$$
W_{a_{\min }, D}^{ \pm}(E)=\tilde{\mathscr{T}}_{\perp}(0) W_{a_{\min }, D}^{ \pm}(0) \tilde{\mathscr{T}}_{\perp}(0)^{*}=e^{i M \alpha_{\perp} \cdot x_{\mathrm{cm}, \perp}} W_{a_{\min }, D}^{ \pm}(0) e^{-i M \alpha_{\perp} \cdot x_{\mathrm{cm}, \perp}},
$$

Theorem 5 can be shown in the same way as above (see also Kiyose [15]). The Dollard type modifier $e^{-i \int_{0}^{t} V\left(s p_{1, \perp} / m_{1}-s \alpha_{\perp}\right) d s}$ in the definition of $W_{a_{\min }, D}^{ \pm}(E)$ seems quite natural, by taking account of that the guiding center of the second particle, which is the only charged one, drifts with the velocity $\alpha_{\perp}$. Here we note that when $N=1$, the corresponding long-range scattering problem has not been solved yet, as far as we know (see Adachi-Kawamoto [3]). Unlike in the case where $N \geq 2$, in general, $V$ does not commute with the conjugate operator $\tilde{A}=q_{1} E \cdot p_{1, \perp}$ (cf. (13)). For reference, the problem of the asymptotic completeness for $\tilde{H}_{\perp}(0)$ with $N \geq 3$ and long-range interactions has not been solved yet, as far as we know. But, maybe one can show the asymptotic completeness under the additional assumption on smooth $V_{j k}$ 's

$$
\left|\partial_{r}^{\beta} V_{j k}(r)\right| \leq C_{\beta}\langle r\rangle^{-\rho-|\beta|}
$$

with some $\sqrt{3}-1<\rho \leq 1$, by using the arguments of Dereziński [16] and Gérard-Łaba [17]. $\sqrt{3}-1$ is called the so-called Enss number. Hence we may expect that a natural extension of Theorem 5 to the case where $N \geq 3$ is obtained.

\section{Concluding Remarks}

We have considered the case where the homogeneous electric field is independent of $t$ only. Here we will make some remarks on the case where the electric field is strictly dependent on $t$.

In order to make the point at issue clear, we suppose that the space dimension $d$ is two, that the time-dependent electric field $E(t)=\left(E_{1}(t), E_{2}(t)\right) \in C\left(\boldsymbol{R} ; \boldsymbol{R}^{2}\right)$ lies in the plane $\boldsymbol{R}^{2}$, and that the system under consideration has at least one charged particle. Then the free Hamiltonian $\tilde{H}_{0, \perp}(E(t))$ of the system is defined by

$$
\tilde{H}_{0, \perp}(E(t))=\sum_{j=1}^{N}\left(\frac{1}{2 m_{j}} D_{j, \perp}^{2}-q_{j} E(t) \cdot x_{j, \perp}\right)
$$


on $L^{2}\left(\boldsymbol{R}^{2 \times N}\right)$. We denote by $\tilde{U}_{0, \perp}(t, s)$ the propagator generated by $\tilde{H}_{0, \perp}(E(t))$. By using the results of Adachi-Kawamoto [3], one can obtain the following Avron-Herbst type formula for $\tilde{U}_{0, \perp}(t, 0)$ immediately:

$$
\tilde{U}_{0, \perp}(t, 0)=\tilde{\mathscr{T}}_{1, \perp}(t) e^{-i t \tilde{H}_{0, \perp}(0)} \tilde{\mathscr{T}}_{1, \perp}(0)^{*}
$$

with

$$
\begin{aligned}
& \tilde{\mathscr{T}}_{1, \perp}(t)=\prod_{j=1}^{N} \mathscr{T}_{j, \perp}(t), \quad \mathscr{T}_{j, \perp}(t)=e^{-i m_{j} a_{j, \perp}(t)} e^{i m_{j} b_{j, \perp}(t) \cdot x_{j, \perp}} e^{-i c_{j, \perp}(t) \cdot k_{j, \perp}}, \\
& b_{j, \perp}(t)^{\mathrm{T}}=\frac{\omega_{j}}{B} \int_{0}^{t} \hat{R}\left(-\omega_{j}(t-s)\right) E(s)^{\mathrm{T}} d s, \quad c_{j, \perp}(t)=\int_{0}^{t} b_{j, \perp}(s) d s \\
& a_{j, \perp}(t)=\int_{0}^{t}\left\{\frac{1}{2} b_{j, \perp}(s)^{2}+\frac{\omega_{j}}{B} b_{j, \perp}(s) \cdot A\left(c_{j, \perp}(s)\right)\right\} d s \\
& \omega_{j}=\frac{q_{j} B}{m_{j}}, \quad \hat{R}(\varphi)=\left(\begin{array}{c}
\cos \varphi-\sin \varphi \\
\sin \varphi
\end{array}\right) .
\end{aligned}
$$

$\left|\omega_{j}\right|$ is called the Larmor frequency of the $j$-th particle. $\omega_{j} / B$ is equal to the specific charge $q_{j} / m_{j}$. The Avron-Herbst type formula (34) with $N=1$ was already obtained in [3]. The differential equations for $a_{j, \perp}(t), b_{j, \perp}(t)$ and $c_{j, \perp}(t)$ are given as

$$
\begin{aligned}
& \dot{b}_{j, \perp}(t)+\frac{2 \omega_{j}}{B} A\left(b_{j, \perp}(t)\right)=\frac{\omega_{j}}{B} E(t), \quad b_{j, \perp}(0)=0, \\
& \dot{c}_{j, \perp}(t)=b_{j, \perp}(t), \quad c_{j, \perp}(0)=0, \\
& \dot{a}_{j, \perp}(t)=\frac{1}{2} b_{j, \perp}(t)^{2}+\frac{\omega_{j}}{B} b_{j, \perp}(t) \cdot A\left(c_{j, \perp}(t)\right), \quad a_{j, \perp}(0)=0
\end{aligned}
$$

(see [3]). Now we introduce the total Hamiltonian $\tilde{H}_{\perp}(E(t))$ of the system is defined by

$$
\tilde{H}_{\perp}(E(t))=\tilde{H}_{0, \perp}(E(t))+V, \quad V=\sum_{1 \leq j<k \leq N} V_{j k}\left(x_{j, \perp}-x_{k, \perp}\right)
$$

on $L^{2}\left(\boldsymbol{R}^{2 \times N}\right)$, and denote by $\tilde{U}_{\perp}(t, s)$ the propagator generated by $\tilde{H}_{\perp}(E(t))$. Then the following AvronHerbst type formula for $\tilde{U}_{\perp}(t, 0)$ can be obtained by virtue of $(34)$ :

Theorem 6 Denote by $\bar{U}_{\perp}(t, s)$ the propagator generated by the time-dependent Hamiltonian

$$
\begin{aligned}
& \bar{H}_{\perp}(t)=\tilde{H}_{0, \perp}(0)+V(t) \\
& V(t)=\sum_{1 \leq j<k \leq N} V_{j k}\left(\left(x_{j, \perp}+c_{j, \perp}(t)\right)-\left(x_{k, \perp}+c_{k, \perp}(t)\right)\right)
\end{aligned}
$$

on $L^{2}\left(\boldsymbol{R}^{2 \times N}\right)$. Suppose that both $\tilde{U}_{\perp}(t, s)$ and $\bar{U}_{\perp}(t, s)$ exist uniquely. Then the Avron-Herbst type formula

$$
\tilde{U}_{\perp}(t, 0)=\tilde{\mathscr{T}}_{1, \perp}(t) \bar{U}_{\perp}(t, 0) \tilde{\mathscr{T}}_{1, \perp}(0)^{*}
$$

holds.

We note $\tilde{\mathscr{T}}_{1, \perp}(0)=\mathrm{Id}$, because $\mathscr{T}_{j, \perp}(0)=\mathrm{Id}$ for any $j$. By definition, if the specific charges $q_{j} / m_{j}$ and $q_{k} / m_{k}$ are different from each other, then $b_{j, \perp}(t) \neq b_{k, \perp}(t)$ and $c_{j, \perp}(t) \neq c_{k, \perp}(t)$ in general, because $\omega_{j} \neq \omega_{k}$. Hence $V(t)$ is time-dependent generally. Because of this, it seems hard to get useful propagation properties of $\bar{U}_{\perp}(t, 0)$. To overcome this difficulty is an issue in the future. However, if all the specific charges are the same, then $b_{1, \perp}(t)=\cdots=b_{N, \perp}(t)$ and $c_{1, \perp}(t)=\cdots=c_{N, \perp}(t)$ hold. Then $V(t)$ is time-independent. Hence we have the following corollary: 
Corollary 7 Suppose that all the specific charges of the system are the same. Then the Avron-Herbst type formula

$$
\begin{aligned}
& \tilde{U}_{\perp}(t, 0)=\tilde{\mathscr{T}}_{1, \perp}(t) e^{-i t \tilde{H}_{\perp}(0)} \tilde{\mathscr{T}}_{1, \perp}(0)^{*} \\
& \tilde{\mathscr{T}}_{1, \perp}(t)=e^{-i M a_{\text {total }, \perp}(t)} e^{i M b_{\text {total }, \perp}(t) \cdot x_{\mathrm{cm}, \perp}} e^{-i c_{\text {total }, \perp}(t) \cdot k_{\text {total }, \perp}}
\end{aligned}
$$

holds with

$$
\begin{aligned}
& b_{\text {total }, \perp}(t)^{\mathrm{T}}=\frac{\Omega}{B} \int_{0}^{t} \hat{R}(-\Omega(t-s)) E(s)^{\mathrm{T}} d s \\
& c_{\text {total }, \perp}(t)=\int_{0}^{t} b_{\text {total }, \perp}(s) d s \\
& a_{\text {total }, \perp}(t)=\int_{0}^{t}\left\{\frac{1}{2} b_{\text {total }, \perp}(s)^{2}+\frac{\Omega}{B} b_{\text {total }, \perp}(s) \cdot A\left(c_{\text {total }, \perp}(s)\right)\right\} d s \\
& \Omega=\frac{Q B}{M}=\omega_{1}=\cdots=\omega_{N} .
\end{aligned}
$$

In this case, the unique existence of $\tilde{U}_{\perp}(t, s)$ can be guaranteed by the self-adjointness of $\tilde{H}_{\perp}(0)$, in virtue of (40). (40) can be also obtained directly as in [3]. We will give an outline of the proof. Put

$$
\hat{U}_{\perp}(t):=e^{-i M a_{\mathrm{total}, \perp}(t)} e^{i M b_{\mathrm{total}, \perp}(t) \cdot x_{\mathrm{cm}, \perp}} e^{-i c_{\mathrm{total}, \perp}(t) \cdot k_{\mathrm{total}, \perp}} e^{-i t \tilde{H}_{\perp}(0)} .
$$

By differentiating (42) in $t$ formally, one can obtain

$$
\begin{aligned}
i \dot{\hat{U}}_{\perp}(t)=e^{-i M a_{\mathrm{total}, \perp}(t)} e^{i M b_{\mathrm{total}, \perp}(t) \cdot x_{\mathrm{cm}, \perp}} e^{-i c_{\mathrm{total}, \perp}(t) \cdot k_{\mathrm{total}, \perp}} \tilde{H}_{\perp}(0) e^{-i t \tilde{H}_{\perp}(0)} \\
+e^{-i M a_{\mathrm{total}, \perp}(t)} e^{i M b_{\mathrm{total}, \perp}(t) \cdot x_{\mathrm{cm}, \perp}} e^{-i c_{\mathrm{total}, \perp}(t) \cdot Q A\left(x_{\mathrm{cc}, \perp}\right)} \\
\quad \times\left(\dot{c}_{\mathrm{total}, \perp}(t) \cdot p_{\mathrm{total}, \perp}\right) e^{-i c_{\mathrm{total}, \perp}(t) \cdot p_{\mathrm{total}, \perp}} e^{-i t \tilde{H}_{\perp}(0)} \\
\quad+\left(M \dot{a}_{\mathrm{total}, \perp}(t)-M \dot{b}_{\mathrm{total}, \perp}(t) \cdot x_{\mathrm{cm}, \perp}+\dot{c}_{\mathrm{total}, \perp}(t) \cdot Q A\left(x_{\mathrm{cc}, \perp}\right)\right) \hat{U}_{\perp}(t) .
\end{aligned}
$$

Here we used

$$
e^{-i c_{\text {total }, \perp}(t) \cdot k_{\text {total }, \perp}}=e^{-i c_{\text {total }, \perp}(t) \cdot Q A\left(x_{\mathrm{cc}, \perp}\right)} e^{-i c_{\text {total }, \perp}(t) \cdot p_{\text {total }, \perp}} .
$$

Since $\tilde{H}_{\perp}(0)$ commutes with $e^{-i c_{\text {total }, \perp}(t) \cdot k_{\text {total }, \perp}}$

$$
e^{-i c_{\mathrm{total}, \perp}(t) \cdot Q A\left(x_{\mathrm{cc}, \perp}\right)} p_{\mathrm{total}, \perp} e^{i c_{\mathrm{total}, \perp}(t) \cdot Q A\left(x_{\mathrm{cc}, \perp}\right)}=p_{\mathrm{total}, \perp}-Q A\left(c_{\mathrm{total}, \perp}(t)\right),
$$

we have

$$
i \dot{\hat{U}}_{\perp}(t)=\hat{H}_{\perp}(t) \hat{U}_{\perp}(t)
$$

with

$$
\begin{aligned}
\hat{H}_{\perp}(t)=\sum_{j=1}^{N} \frac{1}{2 m_{j}}\left(p_{j, \perp}-m_{j} b_{\text {total }, \perp}(t)-q_{j} A\left(x_{j, \perp}\right)\right)^{2}+V \\
+\dot{c}_{\text {total }, \perp}(t) \cdot\left(p_{\text {total }, \perp}-M b_{\text {total }, \perp}(t)-Q A\left(c_{\text {total }, \perp}(t)\right)\right) \\
+M \dot{a}_{\text {total }, \perp}(t)-M \dot{b}_{\text {total }, \perp}(t) \cdot x_{\mathrm{cm}, \perp}+\dot{c}_{\text {total }, \perp}(t) \cdot Q A\left(x_{\mathrm{cc}, \perp}\right) \\
=\tilde{H}_{\perp}(0)+\left(-b_{\text {total }, \perp}(t)+\dot{c}_{\text {total }, \perp}(t)\right) \cdot\left(p_{\text {total }, \perp}-Q A\left(x_{\mathrm{cc}, \perp}\right)\right) \\
+2 \dot{c}_{\text {total }, \perp}(t) \cdot Q A\left(x_{\mathrm{cc}, \perp}\right)-M \dot{b}_{\text {total }, \perp}(t) \cdot x_{\mathrm{cm}, \perp} \\
+M \dot{a}_{\text {total }, \perp}(t)-\dot{c}_{\text {total }, \perp}(t) \cdot\left(M b_{\text {total }, \perp}(t)+Q A\left(c_{\text {total }, \perp}(t)\right)\right) \\
+\frac{M}{2} b_{\text {total }, \perp}(t)^{2} .
\end{aligned}
$$

If we take $c_{\text {total, } \perp}(t)$ as

$$
\dot{c}_{\text {total }, \perp}(t)=b_{\text {total }, \perp}(t), \quad c_{\text {total }, \perp}(0)=0,
$$


then we have

$$
\begin{aligned}
\hat{H}_{\perp}(t)=\tilde{H}_{\perp}(0)-2 Q A\left(b_{\mathrm{total}, \perp}(t)\right) \cdot x_{\mathrm{cc}, \perp}-M \dot{b}_{\mathrm{total}, \perp}(t) \cdot x_{\mathrm{cm}, \perp} \\
\quad+M \dot{a}_{\mathrm{total}, \perp}(t)-\frac{M}{2} b_{\mathrm{total}, \perp}(t)^{2}-Q b_{\mathrm{total}, \perp}(t) \cdot A\left(c_{\mathrm{total}, \perp}(t)\right) .
\end{aligned}
$$

Here we used $b_{\text {total }, \perp}(t) \cdot A\left(x_{\mathrm{cc}, \perp}\right)=-A\left(b_{\text {total }, \perp}(t)\right) \cdot x_{\mathrm{cc}, \perp}$. Moreover, if we take $a_{\text {total }, \perp}(t)$ as

$$
\dot{a}_{\text {total }, \perp}(t)=\frac{1}{2} b_{\text {total }, \perp}(t)^{2}+\frac{Q}{M} b_{\text {total }, \perp}(t) \cdot A\left(c_{\text {total }, \perp}(t)\right), \quad a_{\text {total }, \perp}(0)=0,
$$

then we have

$$
\hat{H}_{\perp}(t)=\tilde{H}_{\perp}(0)-\left(2 Q A\left(b_{\mathrm{total}, \perp}(t)\right) \cdot x_{\mathrm{cc}, \perp}+M \dot{b}_{\mathrm{total}, \perp}(t) \cdot x_{\mathrm{cm}, \perp}\right) .
$$

If $N=1$, then $x_{\mathrm{cc}, \perp}=x_{\mathrm{cm}, \perp}$ holds automatically (cf. [3]). On the other hand, if $N \geq 2$, then $x_{\mathrm{cc}, \perp} \neq x_{\mathrm{cm}, \perp}$ in general, except in the case where all the specific charges are the same. Since $x_{\mathrm{cc}, \perp}=x_{\mathrm{cm}, \perp}$ by assumption, if we take $b_{\text {total }, \perp}(t)$ as

$$
2 Q A\left(b_{\text {total }, \perp}(t)\right)+M \dot{b}_{\text {total }, \perp}(t)=Q E(t), \quad b_{\text {total }, \perp}(0)=0,
$$

then

$$
\hat{H}_{\perp}(t)=\tilde{H}_{\perp}(E(t))
$$

holds. This yields $\hat{U}_{\perp}(t)=\tilde{U}_{\perp}(t, 0)$. $b_{\text {total }, \perp}(t), c_{\text {total }, \perp}(t)$ and $a_{\text {total }, \perp}(t)$ are given by $(41)$ as in [3].

Now, as $E(t)$ under consideration, we take the rotating electric field

$$
E_{\nu, \theta}(t)=E_{0}(\cos (\nu t+\theta), \sin (\nu t+\theta))
$$

with $E_{0}>0, \nu \in \boldsymbol{R}$ and $\theta \in[0,2 \pi)$. Fix $j \in\left\{N_{\mathrm{n}}+1, \ldots, N\right\}$, that is, $j \in\{1, \ldots, N\}$ such that $q_{j} \neq 0$. If $\nu=0$, that is, $E(t) \equiv E_{0}(\cos \theta, \sin \theta)$, then $c_{j, \perp}(t)-t \alpha_{\perp}$ is bounded in $t$, where

$$
\alpha_{\perp}=\frac{E_{0}}{B}(\sin \theta,-\cos \theta)
$$

is the drift velocity. If $\nu=-\omega_{j}$, then $c_{j, \perp}(t)-\left(-t \alpha_{j, \perp}(t)\right)$ is bounded in $t$, where

$$
\alpha_{j, \perp}(t)=\frac{E_{0}}{B}\left(\sin \left(-\omega_{j} t+\theta\right),-\cos \left(-\omega_{j} t+\theta\right)\right)
$$

is the instantaneous drift velocity. Here we note that

$$
\left|\alpha_{\perp}\right|=\left|\alpha_{j, \perp}(t)\right|=\frac{E_{0}}{B}
$$

Hence, in both two cases, the growth order of $\left|c_{j, \perp}(t)\right|$ is $O(|t|)$ as $|t| \rightarrow \infty$. The case where $\nu=-\omega_{j}$ is closely related to the so-called cyclotron resonance. On the other hand, if $\nu \neq 0$ and $\nu \neq-\omega_{j}$, then $c_{j, \perp}(t)$ is bounded in $t$. These results are due to [3]. Here we suppose that $N=2$, that the first particle is charged, and that the specific charge of the second particle is different from that of the first one. Let $\nu=-\omega_{1}$. Then, by virtue of the above results, we see that the growth order of $\left|c_{1, \perp}(t)-c_{2, \perp}(t)\right|$ is $O(|t|)$ as $|t| \rightarrow \infty$, which implies the possibility of the existence of scattering states for the system. Roughly speaking, by virtue of the effect of the cyclotron resonance, the separation of these two particles may occur. In fact, Sato [18] showed the existence of (modified) wave operators under some appropriate assumption on $V_{12}$, because some useful propagation properties of the free propagator $\tilde{U}_{0, \perp}(t, 0)$ can be obtained by using (34) and the argument of [3]. On the other hand, the problem of the asymptotic completeness for such a system has not been solved yet. To get some useful propagation properties of $\tilde{U}_{\perp}(t, 0)$ is an issue in the future.

Acknowledgments. The first author is partially supported by the Grant-in-Aid for Scientific Research (C) \#17K05319 from JSPS. 


\section{References}

1. C. Gérard and I. Łaba, Multiparticle quantum scattering in constant magnetic fields. American Mathematical Society, 2002.

2. E. Skibsted, "Asymptotic completeness for particles in combined constant electric and magnetic fields, II," Duke Math. J., vol. 89, no. 2, pp. 307-350, 1997.

3. T. Adachi and M. Kawamoto, "Avron-Herbst type formula in crossed constant magnetic and time-dependent electric fields," Lett. Math. Phys., vol. 102, no. 1, pp. 65-90, 2012.

4. J. Chee, "Landau problem with a general time-dependent electric field," Ann. Physics, vol. 324, no. 1, pp. 97-105, 2009.

5. X. P. Wang, "On the magnetic Stark resonances in two-dimensional case," in Lecture Notes in Phys., 403. Springer, 1992, pp. 211-233.

6. M. Dimassi and V. Petkov, "Resonances for magnetic Stark Hamiltonians in two-dimensional case," Int. Math. Res. Not., vol. 2004, no. 77, pp. 4147-4179, 2004.

7. — - "Spectral shift function for operators with crossed magnetic and electric fields," Rev. Math. Phys., vol. 22, no. 4, pp. 355-380, 2010.

8. _ - "Spectral problems for operators with crossed magnetic and electric fields," J. Phys. A, vol. 43, no. 47, 474015 (14pp.), 2010.

9. C. Ferrari and H. Kovařŕk, "Resonance width in crossed electric and magnetic field," J. Phys. A, vol. 37, no. 31, pp. 7671-7697, 2004.

10. _ - "On the exponential decay of magnetic Stark resonances," Rep. Math. Phys., vol. 56, no. 2, pp. 197-207, 2005.

11. M. Kawamoto, "Exponential decay property for eigenfunctions of Landau-Stark Hamiltonian," Rep. Math. Phys., vol. 77, no. 1, pp. 129-140, 2016.

12. L. M. Lawson and G. Y. H. Avossevou, "Landau problem with time dependent mass in time dependent electric and harmonic background fields," J. Math. Phys., vol. 59, no. 4, 042109 (9pp.), 2018.

13. T. Adachi, "Scattering theory for a two-body quantum system in a constant magnetic field," J. Math. Sci. Univ. Tokyo, vol. 8, no. 2, pp. 243-274, 2001.

14. —— "On spectral and scattering theory for $N$-body Schrödinger operators in a constant magnetic field," Rev. Math. Phys., vol. 14, no. 2, pp. 199-240, 2002.

15. A. Kiyose, "Scattering theory for two-body quantum systems in crossed constant magnetic and electric fields (in Japanese)," Master's thesis, Kobe University, 2014.

16. J. Dereziński, "Asymptotic completeness of long-range N-body quantum systems," Ann. of Math. (2), vol. 138, no. 2, pp. 427-476, 1993.

17. C. Gérard and I. Łaba, "Scattering theory for $N$-particle systems in constant magnetic fields, II. Long-range interactions," Comm. Partial Differential Equations, vol. 20, no. 9-10, pp. 1791-1830, 1995.

18. Y. Sato, "On two-body scattering in the plane to which crossed constant magnetic and rotating electric fields are impressed (in Japanese)," Master's thesis, Kobe University, 2017. 